\title{
Role of intraoperative dynamic magnetic resonance myelogram in management of giant dorsolumbar spinal extradural arachnoid cyst: case report
}

\author{
Rakesh Kumar Mishra, MBBS, ${ }^{1}$ Nupur Pruthi, MCh, ${ }^{1}$ Rose Dawn Bharath, DM Neuroradiology, ${ }^{2}$ and \\ Bhaskara Rao Malla, DNB, FRCS ${ }^{1}$
}

\begin{abstract}
Departments of ${ }^{1}$ Neurosurgery and ${ }^{2}$ Neuroimaging and Interventional Radiology, National Institute of Mental Health and Neurosciences, Bengaluru, India
\end{abstract}

\begin{abstract}
Giant dorsolumbar spinal arachnoid cysts are a complex, poorly understood, and difficult to manage clinical entity. Traditional CT myelography is technically difficult to use in these cases to detect the site of leakage preoperatively. The authors report a novel technique for detecting the site of the leak by using sequential, dynamic intraoperative MR myelography. To the authors' knowledge, there is no other similar report in the literature.

https://thejns.org/doi/abs/10.3171/2017.2.SPINE16637

KEY WORDS dynamic magnetic resonance myelogram; giant spinal arachnoid cyst; extradural arachnoid cyst; intraoperative magnetic resonance myelogram; dural defect; intrathecal gadolinium; congenital
\end{abstract}

$\mathrm{T}$ HE term "spinal arachnoid cyst" denotes a relatively rare entity among spinal lesions. According to some authors, extradural cysts are less common than intradural ones, but others don't believe this. Symptomatic extradural cysts are even less common. Preoperative detection of the dural defect is critical in managing these lesions. This is done with investigative modalities like CT myelography in most of the cases. Giant spinal extradural arachnoid cysts extending to the sacral levels present a unique difficulty - no space is available to do traditional CT myelography. This problem can be obviated with the use of intraoperative dynamic MR myelography. There are various surgical approaches for management of spinal arachnoid cysts. The principle of treatment in the spinal extradural arachnoid cyst is to do all possible investigations to find the site of communication between the intradural compartment and the cyst, and this is usually done by myelography. Giant dorsal lumbar arachnoid cysts are even more difficult to treat because myelograms are difficult to obtain in all cases. The traditional approach of laminectomy and marsupialization has certain disadvantages that include a high recurrence rate, back pain, and kyphosis. To our knowledge, we are reporting the first case of the use of dynamic MRI in the operative management of a spinal arachnoid cyst.

\section{Case Report}

History and Examination

A 25-year-old woman presented with chief symptoms of middle- and low-back pain, numbness below the waist, weakness in both lower limbs, and a burning sensation in both lower limbs for 10 years. Her symptoms had been worsening over the last 6 months, with the onset of walking difficulty, which progressed to paraplegia and a bedbound stage in the last 5 months. There was no history of a sensation of stiffness in any of the limbs, difficulty in walking in the dark or over uneven surfaces, or bowel or bladder disturbances. There were no other contributing medical or surgical comorbidities. There were no significant contributory general or systemic findings.

Evaluation of the nervous system revealed the patient to be conscious; obeying commands; oriented to place, person, and time; and alert, with normal speech and memory. 
Cranial nerve functions were normal. Muscle bulk and tone was normal in her upper limbs and decreased in bilateral lower limbs. According to the Medical Research Council (MRC) scale, power was $5 / 5$ in all muscle groups of the upper limbs. ${ }^{6}$ On examination of bilateral lower limbs, bilateral hip flexion was $3 / 5$, and extension, abduction, and adduction were $2 / 5$. Right knee flexion was $2 / 5$, extension was $2 / 5$, left knee flexion was $3 / 5$, and extension was $0 / 5$. Right ankle flexion was $0 / 5$, left ankle dorsiflexion was $2 / 5$, right toes were $0 / 5$, and left toes were $2 / 5$. All deep tendon reflexes (DTRs) were 2+, and bilateral ankle and knee jerks were absent. Abdominal reflex was absent, plantar reflex had a bilateral mute response. Sensory examination revealed graded sensory loss below T-10. Bilateral lower-limb joint position sensations were impaired, whereas perineal sensations were preserved. There were no cerebellar signs, and the patient's gait could not be tested. The Nurick score, was Grade V.? Results of a hemogram, biochemistry, and other laboratory investigations were within normal limits.

The patient was informed of the potential risks of this procedure and the fact that its full safety profile was unknown. She was further informed of and consented to the off-label use of intrathecal Gd.

\section{Neuroimaging}

Admission MRI of the whole spine revealed a well-defined extradural cystic lesion extending from T-10 to S-1, with features suggestive of arachnoid cyst (Fig. 1A). The lesion was compressing the cauda equina, was displaced anteriorly, and showed no enhancement on contrast.

\section{Operation}

First Stage. After discussion with the patient and her relatives on the nature of this disease, management options, and likely complications, surgery was planned for the patient. Surgery was performed in 2 stages. In the first stage, under general anesthesia and in the prone position, the patient underwent a T-8 laminectomy using the C-arm because this vertebra was clearly above the upper margin of the lesion. A lumbar drain was inserted, directed downward. The patient was then moved to the MRI suite under general anesthesia. The patient was transferred prone onto the MRI table following all aseptic precautions. Initial imaging was performed for finalization of the sequence parameters and localization of the catheter. Subsequently, without changing the patient's position or scanner location, $3 \mathrm{ml}$ of Gd (Magnevist) was injected into the intradural catheter through an extension tube.

Serial imaging was done 4 times, until the cyst was seen almost completely filled with contrast on a T1-weighted fast sagittal sequence (TR $835 \mathrm{msec}$, TE $9.6 \mathrm{msec}$, field of view 320 , echo train length 3 , slice thickness $4 \mathrm{~mm}$ ). An axial 3D T1-weighted volumetric interpolated breath-hold examination (VIBE) sequence was also obtained (slice thickness $2 \mathrm{~mm}$, TR $8.7 \mathrm{msec}$, TE $4.7 \mathrm{msec}$, matrix 512 $\times 512$ ) with fat suppression, with a field of view including the surgically treated site and covering the cyst (Fig. 1A). Postcontrast imaging (Fig. 1B and C) revealed progressive filling of the cyst in a craniocaudal direction. The site of the leak was identified along the left root sleeve of L-1 because this was the only site that showed multilobulated irregular morphology (Fig. 1D and E). This additional imaging technique during surgery was critical because in this patient the lesion was extending from T-10 to S-1, restricting the access through the L3/4 space for a conventional CT myelography.

Additionally, because the pressure gradient is from the intradural to the extradural compartment, injecting the contrast through the L3/4 space entailed the chance that it could fill only the cyst without demonstrating the site of the defect. Because the presumed site of the leak was not known, we preferred MRI to CT, considering the radiation dose associated with acquiring the CT scans of the dorsolumbar spine multiple times after addition of contrast. The intraoperative CT that we have is a single slice, and we thought the reconstruction quality might be suboptimal for detecting a small leak.

Second Stage. In the second stage of the surgery, a T12-L2 laminectomy was performed, the defect was identified along the left root sleeve of L-1, and fenestration of the arachnoid cyst was done, with repair of the dural defect (Fig. 1G).

\section{Postoperative Course}

The patient tolerated anesthesia and surgery well. Her postoperative recovery was uneventful, with improvement in pain. Postoperative MRI of the dorsal lumbar spine (Fig. 1F) performed at 6 months showed complete resolution of an arachnoid cyst. The patient was treated postoperatively with supportive medications, physiotherapy, and rehabilitation. She was followed up after 3-month and 6-month intervals. On follow-up, she improved clinically from Nurick Grade V to Grade IV (was able to walk with minimal support). At follow-up the patient had no pain, and her muscle bulk and tone were normal. Power was MRC Grade 4/5 for bilateral hip in flexion, extension, adduction, and abduction; bilateral knee was $4 / 5$ in flexion and other movements in that muscle group; right ankle was $3 / 5$; left ankle was $2 / 5$; right toes were $2 / 5$; and left toes were $2 / 5$. All DTRs were $2+$, and bilateral ankle and knee jerks were absent. Plantar reflex had a mute response bilaterally. The patient had improved significantly in the postoperative neurological examination.

\section{Discussion}

The diagnosis of giant spinal arachnoid cysts is clinical and radiological, with the use of MRI, CT myelography, or a combination of both. ${ }^{8}$ A laminectomy approach can cause postoperative complications, especially when the cyst expands more than one level. Almost all studies highlight the effort to identify the site of the leak by using various imaging modalities. The extradural collection may not correlate with the site of the defect. Identifying the exact location of the defect reduces the extent of laminectomy, marsupialization, and its associated complications.

Ellika et al. studied 5 patients with large extradural arachnoid cysts. All of these fluid collections extended over multiple segments, and MRI demonstrated the fluid collections but not the exact site of the leak. ${ }^{2}$ 

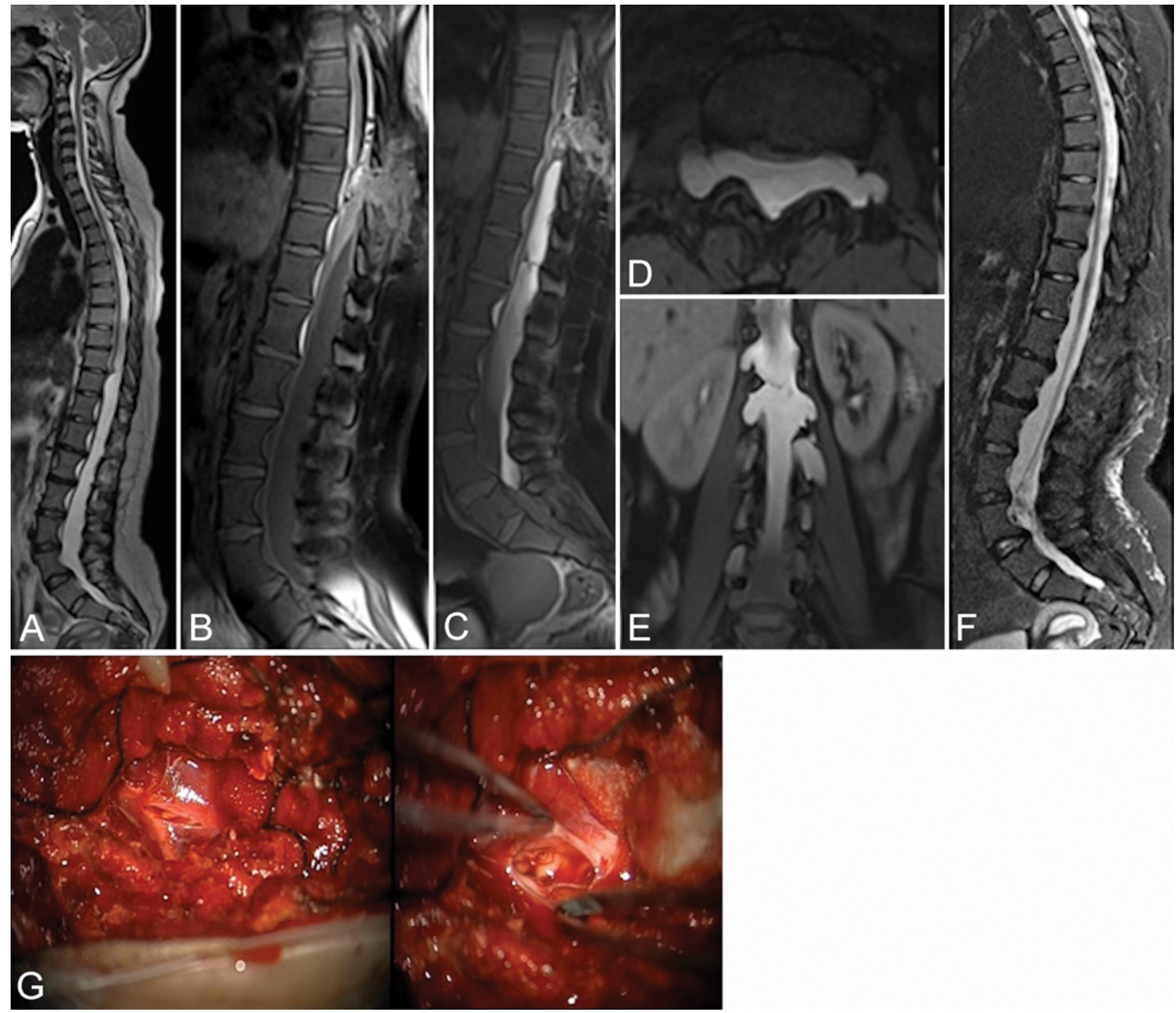

FIG. 1. Midsagittal T2-weighted MRI (A) reveals a large CSF intensity lesion extending from T-10 to S-1, compressing the cauda equina anteriorly. Dynamic intrathecal imaging (B and $\mathbf{C}$ ) reveals sequential filling of the arachnoid cyst superoinferiorly with the dependent layering of contrast. High-resolution 3D T1-weighted VIBE postcontrast axial (D) and coronal (E) imaging reveals foraminal extension of the arachnoid cyst at multiple levels, with a multilobulated appearance at the level of L-1. Postoperative sagittal T2-weighted imaging obtained at 6 months (F) reveals complete resolution of the arachnoid cyst. Intraoperative photograph (G) showing the dural defect along the left root sleeve of L-1. Figure is available in color online only.

Goel et al. reported using Gd-enhanced MR cisternography, and they found it to have potential for clinical applications. They concluded that diagnosis and localization of the fistulous site are better demonstrated due to this modality's high-contrast resolution, the absence of bone artifacts, and direct multiplanar imaging. ${ }^{3}$

We realized that the resolution of the images was the important factor in identifying the site of the defect. Therefore, we performed 3D VIBE sequences in the axial plane in addition to the T1-weighted fast sagittal sequence. The risk profile of administering intrathecal Gd must be considered. Intrathecal Gd is usually found to be safe at lower doses ranging from $0.5 \mathrm{ml}$ to $1 \mathrm{ml}$ in previous studies. ${ }^{4,9}$
Intrathecal Gd is not FDA approved, and serious side effects ranging from nausea, vomiting, headache, anaphylactoid reaction, and seizures to encephalopathy have been reported with the higher dose of Gd administered. ${ }^{5} \mathrm{~Pa}$ tients should, therefore, be made aware of all the potential complications with intrathecal $\mathrm{Gd}$, and special care should be taken to obtain consent from the patients regarding its off-label use. ${ }^{1}$ In our case the patient was moved from the operating theater to the MRI suite, which might create a breach in the sterility. This problem can be avoided by instituting an intraoperative MRI facility and precautions to maintain sterility.

Despite these limitations, we present a novel approach 
in the management of a giant dorsal lumbar arachnoid cyst. The giant spinal extradural arachnoid cyst is a complex and difficult clinical entity. It is difficult to perform traditional CT myelography in such cases. We are reporting a first case in which intraoperative MR myelography has been used for detecting the site of the leak in a giant dorsolumbar arachnoid cyst. We can avoid potential complications of long, multilevel laminectomy and marsupialization. Sequential intraoperative MR myelography is a very useful technique to identify the site of the defect in giant spinal extradural arachnoid cysts, thus reducing the extent of laminectomy and associated morbidity. Using dynamic MR myelography may be especially helpful in centers in which a facility for intraoperative MRI is available.

\section{References}

1. Albayram S, Kilic F, Ozer H, Baghaki S, Kocer N, Islak C: Gadolinium-enhanced MR cisternography to evaluate dural leaks in intracranial hypotension syndrome. AJNR Am J Neuroradiol 29:116-121, 2008

2. Ellika S, Marin H, Pace M, Newman D, Abdulhak M, Kole M: Case Series: Long segment extra-arachnoid fluid collections: Role of dynamic CT myelography in diagnosis and treatment planning. Indian J Radiol Imaging 22:108-115, 2012

3. Goel G, Ravishankar S, Jayakumar PN, Vasudev MK, Shivshankar JJ, Rose D, et al: Intrathecal gadolinium-enhanced magnetic resonance cisternography in cerebrospinal fluid rhinorrhea: road ahead? J Neurotrauma 24:1570-1575, 2007

4. Jinkins JR, Rudwan M, Krumina G, Tali ET: Intrathecal gadolinium-enhanced MR cisternography in the evaluation of clinically suspected cerebrospinal fluid rhinorrhea in humans: early experience. Radiology 222:555-559, 2002

5. Kapoor R, Liu J, Devasenapathy A, Gordin V: Gadolinium encephalopathy after intrathecal gadolinium injection. Pain Physician 13:E321-E326, 2010
6. Medical Research Council: Aids to the Examination of the Peripheral Nervous System. Memorandum No. 45. London: Her Majesty's Stationery Office, 1981

7. Nurick S: The pathogenesis of the spinal cord disorder associated with cervical spondylosis. Brain 95:87-100, 1972

8. Petridis AK, Doukas A, Barth H, Mehdorn HM: Spinal cord compression caused by idiopathic intradural arachnoid cysts of the spine: review of the literature and illustrated case. Eur Spine J 19 (Suppl 2):S124-S129, 2010

9. Tali ET, Ercan N, Krumina G, Rudwan M, Mironov A, Zeng QY, et al: Intrathecal gadolinium (gadopentetate dimeglumine) enhanced magnetic resonance myelography and cisternography: results of a multicenter study. Invest Radiol 37:152-159, 2002

\section{Disclosures}

The authors report no conflict of interest concerning the materials or methods used in this study or the findings specified in this paper.

\section{Author Contributions}

Conception and design: Pruthi, Bharath. Acquisition of data: Mishra. Analysis and interpretation of data: Mishra. Drafting the article: Mishra. Critically revising the article: all authors. Reviewed submitted version of manuscript: all authors. Approved the final version of the manuscript on behalf of all authors: Pruthi. Administrative/technical/material support: Pruthi, Bharath, Malla. Study supervision: Pruthi, Bharath, Malla. Performed the surgery: Pruthi. Performed the MR myelogram: Bharath.

\section{Correspondence}

Nupur Pruthi, Department of Neurosurgery, National Institute of Mental Health \& Neurosciences (NIMHANS), Institute of National Importance, Hosur Rd., Bengaluru, Pin Code 560029, Karnataka, India. email: pruthi_nupur@yahoo.co.in. 